\title{
Ehrlichiosis and Anaplasmosis among Transfusion and Transplant Recipients in the United States
}

\author{
Sanjida J. Mowla, Naomi A. Drexler, Cara C. Cherry, Pallavi D. Annambholta, \\ Ian T. Kracalik, Sridhar V. Basavaraju
}

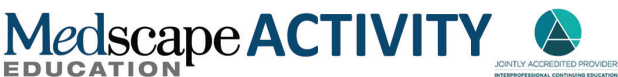

In support of improving patient care, this activity has been planned and implemented by Medscape, LLC and Emerging Infectious Diseases. Medscape, LLC is jointly accredited by the Accreditation Council for Continuing Medical Education (ACCME), the Accreditation Council for Pharmacy Education (ACPE), and the American Nurses Credentialing Center (ANCC), to provide continuing education for the healthcare team.

Medscape, LLC designates this Journal-based CME activity for a maximum of 1.00 AMA PRA Category 1 Credit(s) ${ }^{\text {TM }}$. Physicians should claim only the credit commensurate with the extent of their participation in the activity.

Successful completion of this CME activity, which includes participation in the evaluation component, enables the participant to earn up to $1.0 \mathrm{MOC}$ points in the American Board of Internal Medicine's (ABIM) Maintenance of Certification (MOC) program. Participants will earn MOC points equivalent to the amount of CME credits claimed for the activity. It is the CME activity provider's responsibility to submit participant completion information to ACCME for the purpose of granting ABIM MOC credit.

All other clinicians completing this activity will be issued a certificate of participation. To participate in this journal CME activity: (1) review the learning objectives and author disclosures; (2) study the education content; (3) take the post-test with a 75\% minimum passing score and complete the evaluation at https://www.medscape.org/journal/eid; and (4) view/print certificate.

Release date: October 13, 2021; Expiration date: October 13, 2022

\section{Learning Objectives}

Upon completion of this activity, participants will be able to:

- $\quad$ Assess donor-derived ehrlichiosis and anaplasmosis cases in the United States among solid organ transplant recipients, based on a case series, review of Centers for Disease Control and Prevention (CDC) investigations, and literature review

- Evaluate donor-derived ehrlichiosis and anaplasmosis cases in the United States among transfusion recipients, based on a case series, review of CDC investigations, and literature review

- $\quad$ Determine the clinical implications of donor-derived ehrlichiosis and anaplasmosis cases in the United States among solid organ transplant and transfusion recipients, based on a case series, review of CDC investigations, and literature review

\section{CME Editor}

Tony Pearson-Clarke, MS, Technical Writer/Editor, Emerging Infectious Diseases. Disclosure: Tony Pearson-Clarke, MS, has disclosed no relevant financial relationships.

\section{CME Author}

Laurie Barclay, MD, freelance writer and reviewer, Medscape, LLC. Disclosure: Laurie Barclay, MD, has disclosed no relevant financial relationships.

\section{Authors}

Disclosures: Sanjida J. Mowla, MPH; Naomi A. Drexler, MPH, DrPH; Cara C. Cherry, DVM; Pallavi Annambholta, DrPH; lan T. Kracalik, PhD; and Sridhar V. Basavaraju, MD, have disclosed no relevant financial relationships. 
Ehrlichiosis and anaplasmosis are emerging tickborne diseases that can also be transmitted through blood transfusions or organ transplants. Since 2000, ehrlichiosis and anaplasmosis cases in the United States have increased substantially, resulting in potential risk to transplant and transfusion recipients. We reviewed ehrlichiosis and anaplasmosis cases among blood transfusion and solid organ transplant recipients in the United States from peer-reviewed literature and Centers for Disease Control and Prevention investigations. We identified 132 cases during 1997-2020, 12 transfusionassociated cases and 120 cases in transplant recipients; 8 cases were donor-derived, and in 13 cases illness occurred $<1$ year after transplant. Disease in the remaining 99 cases occurred $\geq 1$ year after transplant, suggesting donor-derived disease was unlikely. Severe illness or death were reported among 15 transfusion and transplant recipients. Clinicians should be alert for these possible infections among transfusion and transplant recipients to prevent severe complications or death by quickly treating them.

E hrlichiosis and anaplasmosis are emerging tickborne diseases caused by Ehrlichia and Anaplasma spp. obligate intracellular bacteria $(1,2)$. Tick bites are the primary route of infection, but transmission can also occur through blood transfusion or solid organ transplantation, because these pathogens infect leukocytes and circulate throughout the blood stream (2). In the United States, human ehrlichiosis is caused primarily by Ehrlichia chaffeensis but can also result from E. ewingii or E. muris eauclairensis infections $(1,3)$. Anaplasmosis is caused by Anaplasma phagocytophilum (1). Although distinct diseases, ehrlichiosis and anaplasmosis share clinical and laboratory features. Early symptoms often include fever, chills, headache, malaise, myalgia, or nausea, and many infections go unrecognized and undiagnosed (3-5). Laboratory features often include leukopenia, thrombocytopenia, anemia, and elevated hepatic transaminases (3-5).

Both diseases have incubation periods of 5-14 days from the time of tick transmission, and during early illness infected asymptomatic persons or those with mild illness might be unknowingly accepted as blood donors $(3,5)$. In general, higher rates of ehrlichiosis and anaplasmosis are reported among adults $>40$ years of age, and most patients are men (5-7). Illness onset is most commonly reported during June and July, corresponding to peak tick activity $(3,5)$. Approximately half of ehrlichiosis and anaplasmosis patients require hospitalization, and $7 \%$ require critical care $(3,8)$. Case-fatality rates are $\approx 1 \%$ for $E$. chaffeensis ehrlichiosis and $0.3 \%$ for anaplasmosis patients based on national surveillance reports $(3,5)$. In part because of immunosuppressive therapies to prevent organ rejection, transplant and transfusion recipients may be more susceptible to ehrlichiosis and anaplasmosis. Relative risk (RR) for severe outcomes among immunosuppressed compared with immunocompetent case-patients was higher for hospitalization (RR 1.4), life-threatening complications (RR 2.4), and death (RR 2.3), highlighting the potential severity of disease in immunocompromised populations $(3,9-15)$.

In the United States, ehrlichiosis was first reported in 1987 and anaplasmosis in 1994, and both became nationally notifiable diseases in $1999(3,5,16,17)$. Since 2000 , reported cases of ehrlichiosis and anaplasmosis in the United States have increased substantially. Reported E. chaffeensis ehrlichiosis cases have increased $>10$-fold, from 200 in 2000 to 2,093 in 2019 (18,19). Reported anaplasmosis cases increased $>16$-fold, from 348 cases in 2000 to 5,655 in $2019(19,20)$.

Increasing rates of reported ehrlichiosis and anaplasmosis might be related to several factors, including improved diagnostics, changes in reporting practices, and expanded human contact with animal reservoirs and tick vectors $(2,3,21,22)$. E. chaffeensis and E. ewingii are primarily transmitted by the lone star tick (Amblyomma americanum); A. phagocytophilum is transmitted by either the blacklegged tick (Ixodes scapularis) or the western blacklegged tick (I. pacificus) $(3,5)$. E. muris eauclaurensis is transmitted by I. scapularis ticks. E. chaffeensis ehrlichiosis is most frequently reported in the southeastern and south-central regions of the United States, and anaplasmosis is most often reported in the upper midwestern and northeastern regions.

PCR and serologic testing using an indirect immunofluorescence antibody assay are the primary laboratory methods for diagnosing ehrlichiosis and anaplasmosis $(3,5)$. Because infection transmitted through blood or organs is rare, it might not be diagnosed in solid organ transplant and transfusion recipients. In addition, nonspecific signs and symptoms and a higher index of suspicion for other opportunistic infections might complicate diagnosis (14), which is unfortunate because early detection and treatment can prevent severe illness and death (23). Here, we summarize and discuss the risks of ehrlichiosis and anaplasmosis cases in the United States among solid organ transplant and transfusion recipients, with a focus on donor-derived infections.

\section{Methods}

We conducted a literature search to identify articles published during January-August 2020 describing ehrlichiosis and anaplasmosis in solid organ 
transplant or blood transfusion recipients in the United States. We used PubMed search terms "ehrlichiosis AND transfusion," "ehrlichiosis AND transplant," "anaplasmosis AND transfusion," and "anaplasmosis AND transplant." We included articles describing case reports, case series, or other clinical descriptions related to Ehrlichia and Anaplasma spp. infections in the setting of solid organ transplantation or transfusion of blood products in the United States. We excluded infections in hematopoietic stem cell recipients because these are not reported to a national notifiable disease system. For articles meeting inclusion criteria, we further reviewed references to identify any case reports or case descriptions not found in the initial PubMed search. Duplicate cases were only counted once for this review. We summarized transplant- and transfusion-associated cases as presented in the literature; we made no additional exclusions based on diagnostic criteria, and we only determined cases to be donor-derived if the authors presented them as such in the literature or investigations.

In the United States, all suspected or confirmed cases of donor-derived diseases are reported to the Organ Procurement and Transplantation Network and are investigated by the Disease Transmission Advisory Committee (DTAC). Nationally notifiable diseases such as ehrlichiosis and anaplasmosis are referred to the Centers for Disease Control and Prevention (CDC) for investigation and determination of whether the infection was transmitted from a donor to a recipient. We also included cases of transplantassociated ehrlichiosis and anaplasmosis identified as part of these DTAC investigations by CDC if not already accounted for in the peer-reviewed literature (Figure 1). From each reviewed article or CDC-led case investigation, we collected available information on geographic region, recipient characteristics, Ehrlichia or Anaplasma species, transmission route, age of blood component transfused or type of organ transplanted, time between transplantation and infection, symptoms and clinical details, diagnostic methods, donor and recipient histories of tick exposure, donor characteristics, likely source of infection, and whether the recipient survived or died.

\section{Results}

\section{Ehrlichiosis and Anaplasmosis Cases Reported among Transfusion Recipients}

During the 1997-2020 investigation period, 12 cases of transfusion-transmitted ehrlichiosis or anaplasmosis were reported in the United States (Appendix Table 1,

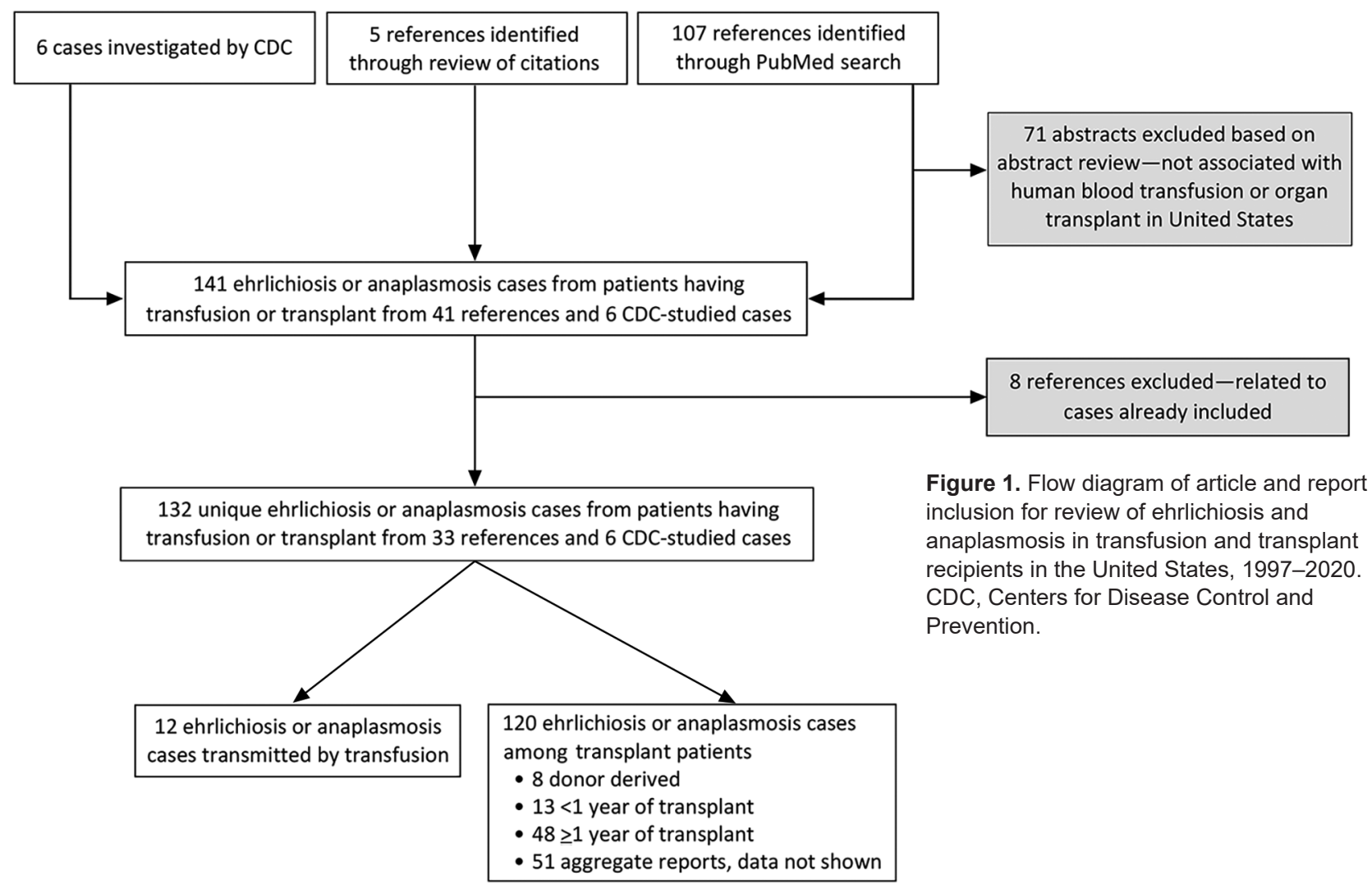


https://wwwnc.cdc.gov/EID/article/27/11/211127-App1.pdf). Of the 12 transfusion-transmitted cases, 8 resulted from transfused red blood cell components and 3 from transfused platelet components ( 2 apheresis and 1 whole blood-derived); the component for 1 case was not identified. Ten $(83.3 \%)$ of 12 transfusion-associated cases were A. phagocytophilum infections; 1 case was associated with $E$. ewingii and 1 with E. chaffeensis (Figure 2, panels C, D). Median age of transfusion recipients was 66 years (range 9-85 years); sex was equally distributed. Of the transfusion-associated cases of ehrlichiosis and anaplasmosis, 3 occurred in Minnesota; 2 in Wisconsin; and 1 each in Georgia, Rhode Island, Connecticut, Massachusetts, New York, and Oklahoma (Figure 2, panels A, B). Disease in all cases was diagnosed using PCR, and additional serologic testing was used for 2 cases. Most $(83.3 \%)$ transfusion case-patients survived infection; one third of cases were associated with mild symptoms. Intensive care unit (ICU) treatment was noted for 2 anaplasmosis patients, prompted by respiratory failure, hypotension, and hypoxia. In addition, 3 anaplasmosis patients had multisystem organ failure, but ICU treatment was not mentioned for these cases. Two patients died, one from anaplasmosis and the other from other medical complications.

\section{Ehrlichiosis and Anaplasmosis Cases Reported among Solid Organ Transplant Recipients}

During the investigation period, 107 cases of ehrlichiosis and 7 cases of anaplasmosis were described in the literature among solid organ transplant recipients. Of these, 63 included patient-specific information. We described the remaining 51 cases in aggregate and reported on them separately. An additional 6 cases of ehrlichiosis among solid organ transplant recipients were investigated by $C D C$, resulting in a total of 120 cases of ehrlichiosis or anaplasmosis described among transplant recipients during the investigation period.

E. chaffeensis ehrlichiosis was the most common organ donor-derived infection, reported among 8 solid organ transplant recipients: 2 cases we identified from the literature and the 6 investigated by CDC (Appendix Table 2). Of these, 7 (87.5\%) case-patients were kidney transplant recipients and 1 was a liver transplant recipient. Fever was reported among all solid organ transplant recipients. Diagnostic methods were PCR $(87.5 \%)$ and serologic testing (12.5\%). Most $(87.5 \%)$ patients were male, median age was 57 years (range 5-69 years), and median time between transplantation and infection was 13.5 days (range 10-25 days). Of the donor-derived ehrlichiosis cases,
2 occurred in Maryland; 3 in Missouri; and 1 each in New York, New Jersey, and Illinois (Figure 2, panel E). Among the 8 donor-derived ehrlichiosis cases, 2 deaths were reported among the kidney transplant recipients, both occurring $<1$ month after transplantation (Appendix Table 3). None of the confirmed transplant-derived case reports described ICU admission among the patients.

Thirteen cases of ehrlichiosis and anaplasmosis occurred $<1$ year after transplantation but could not be confirmed as donor-derived. The median time between transplantation and symptom onset in these 13 cases was 5 months (range 2 weeks- 9 months) (Table 4). Of those cases, 4 were identified in Tennessee (Figure 2, panel F). E. chaffeensis infection was associated with $11(84.6 \%)$ cases, and A. phagocytophilum infection was associated with $2(15.4 \%)$ cases. Of the 13 cases, 5 (38.5\%) occurred in kidney recipients, 4 $(30.8 \%)$ in heart recipients, $2(15.4 \%)$ in kidney and pancreas recipients, and $1(7.7 \%)$ in a liver and lung recipient. Most (76.9\%) patients were male, median age was 50 years (range 35-63 years), and PCR was the most common (76.9\%) diagnostic method. Most $(92.3 \%)$ patients infected $<1$ year after transplantation survived; however, 1 kidney and pancreas recipient with $A$. phagocytophilum infection died.

Forty-eight records of individual cases of ehrlichiosis or anaplasmosis that occurred $\geq 1$ year after transplant were most likely attributable to posttransplant infections acquired through tick bites (Table 5). Median time from transplant to symptom onset was 6 years (range 1-21 years), most patients $(75.0 \%)$ were male, and median age was 51 years (range 11-73 years). Among these patients, records described Ehrlichia infection for $43(89.6 \%)$ and Anaplasma infection for 5 $(10.4 \%)$. E. chaffeensis was implicated in $30(69.8 \%)$ of 43 cases of ehrlichiosis, E. ewingii in 12 (27.9\%), and an unspecified Ehrlichia sp. in 1 (2.3\%). Twenty-three patients $(53.5 \%)$ received kidneys, $8(18.6 \%)$ livers, 7 (16.3\%) lungs, $4(9.3 \%)$ hearts, and $1(2.3 \%)$ a pancreas. Of the 5 reported cases of anaplasmosis occurring $\geq 1$ year after transplant, 4 (80.0\%) occurred in kidney recipients and $1(20.0 \%)$ in a liver recipient. Among the 43 cases of ehrlichiosis in solid organ transplant recipients who had illness onset $\geq 1$ year after transplant, 26 (60.6\%) occurred in Missouri and 12 (27.9\%) in Tennessee; exact location was not specified in 1 report, but the case occurred in Alabama, Tennessee, or Mississippi. Among the 5 cases of anaplasmosis, 2 occurred in Minnesota, 1 in Maine, 1 in Wisconsin, and 1 in Connecticut. One patient had possible reactivation of a previous Anaplasma infection secondary to potent immunosuppression 9 months after the 
original infection. There were 2 reports of Ehrlichia reinfection that described distinct strains of $E$. chaffeensis and $E$. ewingii found in a secondary infection. Secondary hemophagocytic lymphohistiocytosis, which is characterized by severe and potentially fatal inflammation, developed in 1 kidney transplant recipient. Of the solid organ transplant recipients with ehrlichiosis or anaplasmosis occurring $\geq 1$ year after transplant, 47
$(97.9 \%)$ of 48 survived. One pancreas recipient with an $E$. chaffeensis infection died. Seven cases occurring $\geq 1$ year of solid organ transplantation did report ICU admission, possibly relating to long-term immunosuppression among transplant recipients.

Of the 51 cases of ehrlichiosis from Missouri for which we report data in aggregate, 18 (35.3\%) occurred in kidney recipients, 12 (23.5\%) in heart recipients, 12
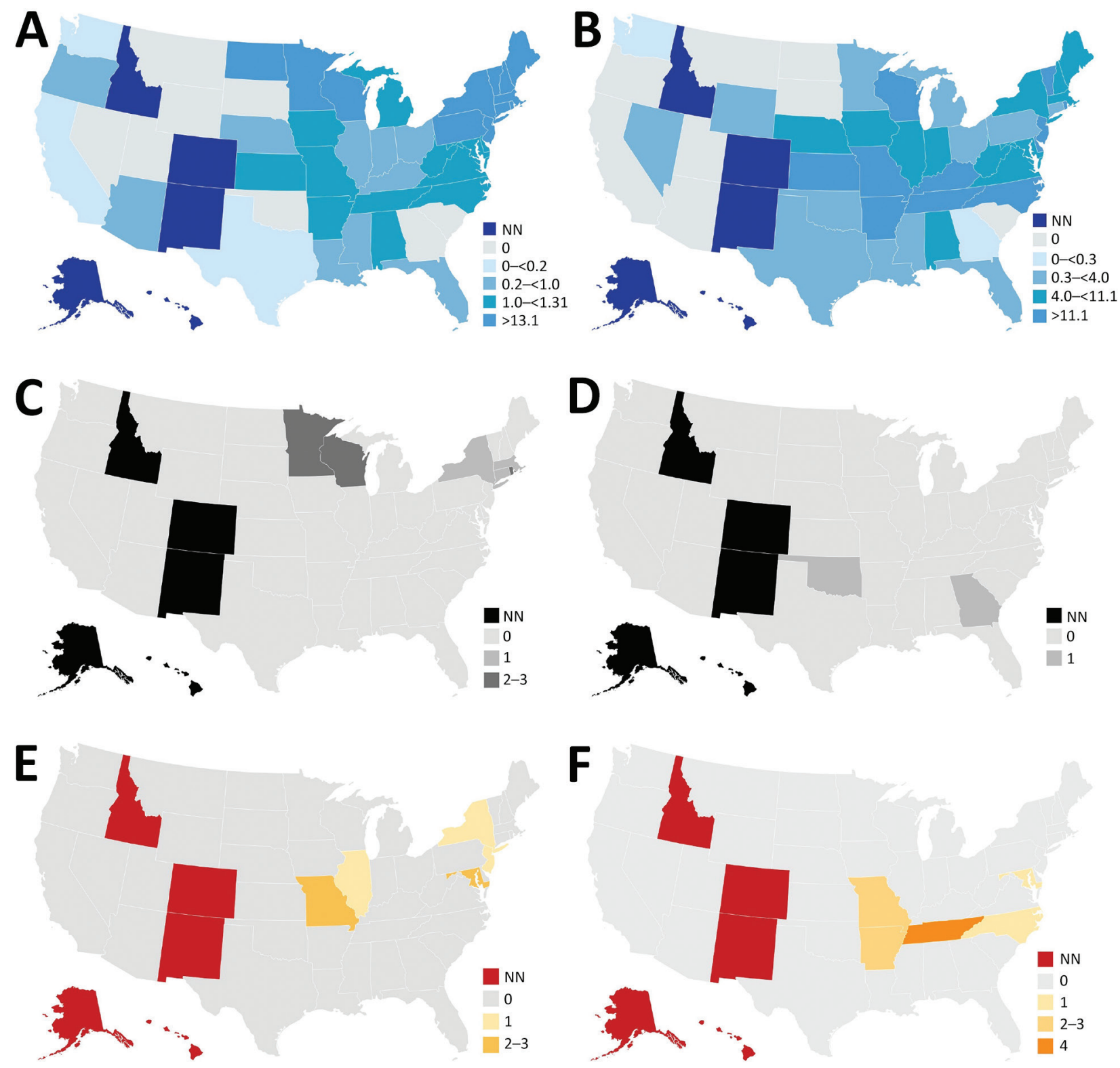

Figure 2. Incidence of ehrlichiosis and anaplasmosis in the United States, 2019, and of cases among transfusion and transplant recipients, 1997-2020. A) Incidence of anaplasmosis per 1 million US residents in 2019. B) Incidence of ehrlichiosis per 1 million US residents in 2019. C) Transfusion-transmitted anaplasmosis cases by recipient state of residence. D) Transfusion-transmitted ehrlichiosis cases by recipient state of residence. E) Organ donor-derived ehrlichiosis cases by recipient state of residence. We identified no organ donor-derived anaplasmosis cases. F) Organ donor-derived ehrlichiosis cases with onset $<1$ year after transplant by recipient state of residence. We identified 2 additional donor-derived ehrlichiosis cases with onset $<1$ year after transplant; however, recipient state of residence was unknown. NN, not notifiable. 
$(23.5 \%)$ in lung recipients, $7(13.7 \%)$ in liver recipients, and $2(4.0 \%)$ in kidney-pancreas recipients. Additional information on disease, pathogen species, and demographics was not available for these 51 cases.

\section{Discussion}

This study reviewed ehrlichiosis and anaplasmosis cases among transfusion and solid organ transplant recipients described in published literature and reports from 6 CDC investigations. During the study period, 12 cases of transfusion-transmitted ehrlichiosis or anaplasmosis were reported, 2 of which resulted in death. In addition, 8 cases of organ donor-derived ehrlichiosis were reported (7 kidney transplant recipients and 1 liver recipient), 2 of which resulted in death. A. phagocytophilum was the most common causative agent among transfusion-derived infections. In contrast, among transplant recipients, E. chaffeensis was the most common causative agent. Donor-derived infections were observed among patients of a broad range of ages (5-85 years). Although children are not considered a high-risk group for anaplasmosis in particular, pediatric infection should not be discounted among transfusion and transplant recipients. Time from transplant or transfusion to the development of signs and symptoms varied widely and in most cases was longer than the typical incubation period for a tick-transmitted infection. Delays in symptom onset might be affected by the colony size and the site of the inoculated bacteria. Transfusion- or transplant-transmitted ehrlichiosis and anaplasmosis are rare but can result in severe outcomes including the death of the recipient.

Although known cases of transfusion- and transplant-transmitted ehrlichiosis or anaplasmosis are uncommon, studies of asymptomatic infection among blood donors and the survivability of infection in blood suggest the risk of transmission is greater than previously recognized. In endemic areas, seroprevalence studies found $11.3 \%$ of blood donors in New York, $0.5 \%$ in Wisconsin, and 3.5\% in Connecticut had detectable antibodies against $A$. phagocytophilum $(24,25)$. In Iowa, $\approx 1 \%$ of blood donors studied were seropositive for and displayed symptoms of ehrlichiosis after blood donation, although recipient lookback reported by physicians indicated that these transfusions did not result in transfusion-transmitted illnesses (26). However, serosurveys do not report active or incident infections, only the proportion of participants previously exposed to an Ehrlichia or Anaplasma agent. Some studies also examined the survivability of Ehrlichia and Anaplasma species in donor blood. Blood from anaplasmosis patients in 2 studies found viable A. phagocytophilum survived under refrigerated storage conditions for up to 18 days in whole blood $(27,28)$. One in vitro study reported $E$. chaffeensis remained viable for up to 11 days within refrigerated packed red blood cells (29).

Donor-derived ehrlichiosis and anaplasmosis highlight the importance of donor risk mitigation strategies in the setting of blood transfusion and solid organ transplantation and the potential role of laboratory-based screening. Recognizing and diagnosing tickborne diseases is complicated by long incubation periods and potential asymptomatic or mildly symptomatic infections. Although several laboratory tests are used to diagnose ehrlichiosis and anaplasmosis, no tests have been approved by the US Food and Drug Administration (FDA) to screen blood or organ donors for these diseases. Furthermore, serologic screening of donors might not identify active Ehrlichia or Anaplasma infections or could exclude healthy donors (28). PCR testing would more accurately screen contaminated blood and organ products, but no FDA-licensed test is available. Donor deferral on the basis of travel or residence would be impractically broad because of the widespread endemicity of ehrlichiosis and anaplasmosis across regions of the United States. To minimize blood supply disruptions based on travel deferrals for Zika virus and babesia, FDA previously recommended universal antibody testing and regional nucleic acid testing in states with the highest rates of risk for infection $(30,31)$. Similar screening may eventually be necessary for ehrlichiosis and anaplasmosis as the prevalence and incidence of these infections increases in the United States. CDC will continue to monitor the occurrence of transfusion- or transplant-transmitted ehrlichiosis and anaplasmosis.

Among this study's limitations, for sources identified through the literature we were limited to the information provided in the case reports, which might not always have represented a full account of the patient experience. Data on donor demographics were extremely limited. Donor state of residence might have provided a more accurate insight on likelihood of donor infection by state which might have broader implications for blood and organ screening criteria. Next, for the purposes of this evaluation, we relied on the characterizations by the original authors or investigators to categorize cases as donoror transplant-derived ehrlichiosis or anaplasmosis. Finally, we included only cases published in peerreviewed literature or reported to CDC, possibly underreporting transfusion- and transplant-associated infections. In 2019, the Rickettsial Zoonoses Branch, 
Division of Vector-Borne Diseases, National Center for Emerging and Zoonotic Infectious Diseases, CDC, added questions relating to recent transfusion and organ transplant to their tickborne rickettsial disease surveillance system (https://www.cdc. gov/ticks/forms/Tick_TBRD_FILL_508.pdf) to better track donor-derived infections.

Because Ehrlichia and Anaplasma species primarily infect leukocytes, leukoreduction has been presumed to reduce risk for ehrlichiosis and anaplasmosis through passive removal $(23,32,33)$. However, $83 \%(10 / 12)$ of components implicated in transfusion-transmitted cases in this study were leukoreduced. In cell culture models, because of their presence in plasma also, E. chaffeensis survived in red blood cells stored in additive solution, suggesting leukoreduction alone will not prevent transmission of Ehrlichia and Anaplasma species (29). Adopting pathogen reduction technology, such as psoralen and ultraviolet light to inactivate infectious agents, for platelet and plasma products would provide additional safety measures to reduce risk for transfusion-transmitted Ehrlichia and Anaplasma infections. Pathogen-reduced plasma has demonstrated a $\geq 3$.6-log reduction in viable $A$. phagocytophilum (34). However, this method has not been approved for red blood cell products, which were implicated in 8 of 12 cases of transfusion-transmitted ehrlichiosis and anaplasmosis in this study.

Identifying risk factors for Ehrlichia and Anaplasma infections among deceased organ donors is challenging because clinical, demographic, and social information about deceased donors is often obtained from family members, who might not have access to or recall all historical details (35). Also, routine laboratory screening of organ donors, required for multiple infectious diseases, including HIV/AIDS, does not yet include testing for infection with Ehrlichia and Anaplasma species. In addition, the number of posttransplant infections reported in this review highlights the risk among blood product or organ recipients. Therefore, clinicians must closely monitor recipients of blood transfusions or solid organs during long-term management and consider these pathogens when recipients develop signs or symptoms of infection. Because the prevalence of tickborne disease infections is rising, additional risk mitigation interventions will likely be necessary to enhance blood and organ safety.

\section{Acknowledgments}

We thank Paige Armstrong for her contributions to preparing this manuscript.

Organ Procurement and Transplantation Network data provided for this report was supported in part by the US Health Resources and Services Administration (https://www.hrsa.gov).

\section{About the Author}

Ms. Mowla is an ORISE fellow in the Office of Blood, Organ, and Other Tissue Safety, Division of Healthcare Quality Promotion, National Center for Emerging and Zoonotic Infectious Diseases, CDC, in Atlanta. Her primary research interests are transfusion- and transplanttransmitted infections.

\section{References}

1 Vannorsdall MD, Thomas S, Smith RP, Zimmerman R, Christman R, Vella JP, et al. Human granulocytic ehrlichiosis in a renal allograft recipient: review of the clinical spectrum of disease in solid organ transplant patients. Transpl Infect Dis. 2002;4:97-101. https:/ / doi.org/10.1034/ j.1399-3062.2002.01015.x

2. Ismail N, Bloch $\mathrm{KC}$, McBride JW. Human ehrlichiosis and anaplasmosis. Clin Lab Med. 2010;30:261-92. https:/ / doi.org/ 10.1016/j.cll.2009.10.004

3. Nichols Heitman K, Dahlgren FS, Drexler NA, Massung RF, Behravesh CB. Increasing incidence of ehrlichiosis in the United States: a summary of national surveillance of Ehrlichia chaffeensis and Ehrlichia ewingii infections in the United States, 2008-2012. Am J Trop Med Hyg. 2016;94:52-60. https://doi.org/10.4269/ajtmh.15-0540

4. Bakken JS, Dumler JS. Human granulocytic anaplasmosis. Infect Dis Clin North Am. 2015;29:341-55. https:/ / doi.org/ 10.1016/j.idc. 2015.02 .007

5. Dahlgren FS, Heitman KN, Drexler NA, Massung RF, Behravesh CB. Human granulocytic anaplasmosis in the United States from 2008 to 2012: a summary of national surveillance data. Am J Trop Med Hyg. 2015;93:66-72. https://doi.org/10.4269/ajtmh.15-0122

6. Fishbein DB, Dawson JE, Robinson LE. Human ehrlichiosis in the United States, 1985 to 1990. Ann Intern Med. 1994;120:736-43. https://doi.org/10.7326/ 0003-4819-120-9-199405010-00003

7. Paddock CD, Childs JE. Ehrlichia chaffeensis: a prototypical emerging pathogen. Clin Microbiol Rev. 2003;16:37-64. https:/ / doi.org/10.1128/CMR.16.1.37-64.2003

8. Dumler JS. Anaplasma and Ehrlichia infection. Ann N Y Acad Sci. 2005;1063:361-73. https://doi.org/10.1196/ annals.1355.069

9. Alhumaidan H, Westley B, Esteva C, Berardi V, Young C, Sweeney J. Transfusion-transmitted anaplasmosis from leukoreduced red blood cells. Transfusion. 2013;53:181-6. https:// doi.org/10.1111/j.1537-2995.2012.03685.x

10. Cotant C, Okulicz JF, Brezina B, Riley DJ, Conger NG. Human monocytic ehrlichiosis in a renal transplant patient. Scand J Infect Dis. 2006;38:699-702. https:/ / doi.org/ $10.1080 / 00365540500444694$ 
11. Fine AB, Sweeney JD, Nixon CP, Knoll BM. Transfusiontransmitted anaplasmosis from a leukoreduced platelet pool. Transfusion. 2016;56:699-704. https:// doi.org/10.1111/ trf.13392

12. Goel R, Westblade LF, Kessler DA, Sfeir M, Slavinski S, Backenson B, et al. Death from transfusion-transmitted anaplasmosis, New York, USA, 2017. [Erratum in Emerg Infect Dis. 2018;24:1773.]. Emerg Infect Dis. 2018;24:1548-50. https://doi.org/10.3201/eid2408.172048

13. Sachdev SH, Joshi V, Cox ER, Amoroso A, Palekar S. Severe life-threatening Ehrlichia chaffeensis infections transmitted through solid organ transplantation. Transpl Infect Dis. 2014;16:119-24. https://doi.org/10.1111/tid.12172

14. Safdar N, Love RB, Maki DG. Severe Ehrlichia chaffeensis infection in a lung transplant recipient: a review of ehrlichiosis in the immunocompromised patient. Emerg Infect Dis. 2002;8:320-3. https://doi.org/10.3201/ eid0803.010249

15. Trofe J, Reddy KS, Stratta RJ, Flax SD, Somerville KT, Alloway RR, et al. Human granulocytic ehrlichiosis in pancreas transplant recipients. Transpl Infect Dis. 2001; 3:34-9. https:// doi.org/10.1034/j.1399-3062.2001.003001034.x

16. Chen SM, Dumler JS, Bakken JS, Walker DH. Identification of a granulocytotropic Ehrlichia species as the etiologic agent of human disease. J Clin Microbiol. 1994;32:589-95. https://doi.org/10.1128/jcm.32.3.589-595.1994

17. Maeda K, Markowitz N, Hawley RC, Ristic M, Cox D, McDade JE. Human infection with Ehrlichia canis, a leukocytic rickettsia. N Engl J Med. 1987;316:853-6. https:/ / doi.org/10.1056/NEJM198704023161406

18. CDC. Ehrlichiosis: epidemiology and statistics [cited 2021 June 23]. https://www.cdc.gov/ehrlichiosis/stats/ index.html

19. CDC. Nationally notifiable infectious diseases and conditions, United States: annual tables [cited 2021 June 23]. https://wonder.cdc.gov/nndss/static/2019/annual/ 2019-table2f.html

20. CDC. Anaplasmosis: epidemiology and statistics [cited 2021 June 23]. https:// www.cdc.gov/anaplasmosis/stats/ index.html

21. Dahlgren FS, Mandel EJ, Krebs JW, Massung RF, McQuiston $\mathrm{JH}$. Increasing incidence of Ehrlichia chaffeensis and Anaplasma phagocytophilum in the United States, 2000-2007. Am J Trop Med Hyg. 2011;85:124-31. https:// doi.org/ 10.4269/ajtmh.2011.10-0613

22. Springer YP, Eisen L, Beati L, James AM, Eisen RJ. Spatial distribution of counties in the continental United States with records of occurrence of Amblyomma americanum (Ixodida: Ixodidae). J Med Entomol. 2014;51:342-51. https:/ / doi.org/ 10.1603/ME13115

23. Biggs HM, Behravesh CB, Bradley KK, Dahlgren FS, Drexler NA, Dumler JS, et al. Diagnosis and management of tickborne rickettsial diseases: Rocky Mountain spotted fever and other spotted fever group rickettsioses, ehrlichioses, and anaplasmosis - United States. A practical guide for health care and public health professionals. MMWR Recomm Rep. 2016;65:1-44. https://doi.org/10.15585/mmwr.rr6502a1

24. Aguero-Rosenfeld ME, Donnarumma L, Zentmaier L, Jacob J, Frey M, Noto R, et al. Seroprevalence of antibodies that react with Anaplasma phagocytophila, the agent of human granulocytic ehrlichiosis, in different populations in Westchester County, New York. J Clin Microbiol. 2002; 40:2612-5. https://doi.org/10.1128/JCM.40.7.2612-2615.2002
25. Leiby DA, Chung AP, Cable RG, Trouern-Trend J, McCullough J, Homer MJ, et al. Relationship between tick bites and the seroprevalence of Babesia microti and Anaplasma phagocytophila (previously Ehrlichia sp.) in blood donors. Transfusion. 2002;42:1585-91. https://doi.org/10.1046/ j.1537-2995.2002.00251.x

26. Arguin PM, Singleton J, Rotz LD, Marston E, Treadwell TA, Slater K, et al. An investigation into the possibility of transmission of tick-borne pathogens via blood transfusion. Transfusion-Associated Tick-Borne Illness Task Force. Transfusion. 1999;39:828-33. https:// doi.org/10.1046/ j.1537-2995.1999.39080828.x

27. Kalantarpour F, Chowdhury I, Wormser GP, Aguero-Rosenfeld ME. Survival of the human granulocytic ehrlichiosis agent under refrigeration conditions. J Clin Microbiol. 2000;38:2398-9. https:/ / doi.org/10.1128/ JCM.38.6.2398-2399.2000

28. McQuiston JH, Childs JE, Chamberland ME, Tabor E. Transmission of tick-borne agents of disease by blood transfusion: a review of known and potential risks in the United States. [Erratum in Transfusion. 2000;40:891.] Transfusion. 2000;40:274-84. https://doi.org/10.1046/ j.1537-2995.2000.40030274.x

29. McKechnie DB, Slater KS, Childs JE, Massung RF, Paddock CD. Survival of Ehrlichia chaffeensis in refrigerated, ADSOL-treated RBCs. Transfusion. 2000;40:1041-7. https://doi.org/10.1046/j.1537-2995.2000.40091041.x

30. Food and Drug Administration. Recommendations for reducing the risk of transfusion-transmitted babesiosis [cited on 2021 June 23]. https:/ / www.fda.gov/media/114847/ download

31. Food and Drug Administration. Information for blood establishments regarding FDA's determination that Zika virus is no longer a relevant transfusion-transmitted infection [cited 2021 September 7]. https:// www.fda.gov/vaccinesblood-biologics/blood-blood-products/information-bloodestablishments-regarding-fdas-determination-zika-virus-nolonger-relevant

32. Annen K, Friedman K, Eshoa C, Horowitz M, Gottschall J, Straus T. Two cases of transfusion-transmitted Anaplasma phagocytophilum. Am J Clin Pathol. 2012;137:562-5. https:/ / doi.org/10.1309/AJCP4E4VQQQOZIAQ

33. Proctor MC, Leiby DA. Do leukoreduction filters passively reduce the transmission risk of human granulocytic anaplasmosis? Transfusion. 2015;55:1242-8. https://doi.org/10.1111/trf.12976

34. Food and Drug Administration. INTERCEPT blood system for cryoprecipitation package insert for the manufacturing of pathogen reduced plasma, cryoprecipitate reduced [cited on 2021 June 23]. https:/ / www.fda.gov/media/90594/ download

35. Vora NM, Basavaraju SV, Feldman KA, Paddock CD, Orciari L, Gitterman S, et al.; Transplant-Associated Rabies Virus Transmission Investigation Team. Raccoon rabies virus variant transmission through solid organ transplantation. JAMA. 2013;310:398-407. https:/ /doi.org/ 10.1001/jama.2013.7986

Address for correspondence: Sridhar V. Basavaraju, Centers for Disease Control and Prevention, 1600 Clifton Rd NE, Mailstop V18-4, Atlanta, GA 30329-4027, USA; email: smowla1@uga.edu 